\title{
Novel Design for Noise Controlled Semiconductor Optical Amplifier
}

\author{
Séverine Philippe ${ }^{1}$, Frederic Surre ${ }^{2}$, Kevin Carney ${ }^{1}$, R. Lennox ${ }^{3}$, A. Louise Bradley ${ }^{3}$, Pascal Landais ${ }^{1}$ \\ 1) Dublin City University, Dublin 9, Ireland \\ 2) City University London, Northampton Square, London, UK \\ 3) Trinity College Dublin, Dublin 2, Ireland \\ Tel: (353)(1) 700 8044, Fax: (353)(1) 7005508, e-mail: landaisp@eeng.dcu.ie
}

\begin{abstract}
The use of semiconductor optical amplifiers (SOA) in optical communications networks has so far been limited due to their inherent large noise figure (NF) compared to Erbium Doped Fibre Amplifiers. Therefore improvement of the noise performance of SOAs is critical to their widespread adoption in future networks. We propose to reduce the NF of the SOA by introducing a lasing cavity lateral to the axis of amplification of the device. The carrier density within the cavity is clamped at the lasing threshold. It is thus possible to engineer the carrier density distribution along the active waveguide by controlling the cavity design. According to our simulations, some of the cavity designs lead to a reduction of the noise figure in this novel SOA.
\end{abstract}

Keywords: Semiconductor optical amplifier, noise, optical communications

\section{INTRODUCTION}

One of the most promising components to become the core of all-optical metropolitan networks is the semiconductor optical amplifier (SOA). Besides acting as an amplifier, its inherent non-linearities allow it to be the basis of many all-optical signal processing functions, usually based on polarisation rotation or gain compression [1,2], such as wavelength converters and logic gates. Compared to Erbium doped fibre amplifier (EDFA), Erbium doped waveguide amplifier (EDWA) or Raman amplifier, SOA has many advantages: lower cost, larger bandwidth, smaller size, potential to be integrated on a chip and electrical pumping. However it has one drawback, a higher noise figure (NF) than these competitors [3]. The NF is linked to excess noise introduced by the amplifier. It corresponds to the ratio between the signal-to-noise ratio at the input and at the output. It is essential to have this NF as low as possible.

Several approaches have been considered to solve this problem. One of them is on the SOA waveguide. It was showed that with a low confinement factor a reduction of the NF could be perceived. The results achieved are encouraging, but the NF of this device is still not as comparable to that of an EDFA [4]. Some laboratories have considered a chain of amplifiers, with a low NF SOA and low gain placed at the input and noisier SOAs with higher gain positioned further down in the amplification scheme. This approach should give some good results, however it does not solve the problem of a low NF SOA [5].

In this paper we propose to achieve a lower noise figure by engineering the carrier density profile along the active region of our device. To this end we employ a lateral lasing cavity perpendicular to the axis of propagation. This is made possible by introducing slots that act as mirrors on both sides of the active layer parallel to the growth direction.

\section{MODEL}

\subsection{Carrier density}

Our model is based on a numerical solution of the travelling wave description of the E-field in the amplifier cavity [6]. The cavity is divided into sub-sections with a constant carrier density and amplified spontaneous emission. The program calculates the variation of the E-field and carrier density in each section along the SOA. The rate of change of the carrier density, $\mathrm{N}_{\mathrm{m}}$, in each section of the SOA is given by:

$$
\frac{d N_{m}}{d t}=\frac{I_{m}}{e V_{m}}-R\left(N_{m}\right)-\left[g\left(\omega, N_{m}\right) S_{m, s i g}+a_{m}\left(N_{m}-N_{0}\right) S_{m, s p o n}\right] \frac{c}{n_{g, 0}},
$$

where $I_{m}$ is the current applied to the $\mathrm{m}^{\text {th }}$ section $V_{m}$, the volume of the active layer of this section, $e$ is the elementary charge, $R(N)$ is the carrier recombination rate, $g$ is the material gain, $S_{m, s i g}$ and $S_{m, s p o n}$ are the average photon densities for the signal and spontaneous emission, $a_{m}$ is the gain coefficient, $N_{0}$ is the carrier density at transparency, $c$ is the celerity of light and $n_{g, 0}$ is the group index.

\subsection{Noise figure}

The noise figure can then be calculated from the carrier density in each section. As the device is considered a cascade of $\mathrm{n}$ smaller amplifiers, with a uniform carrier density, the noise figure is given by Friis' transmission formula as: 


$$
N F_{\text {total }}=N F_{1}+\frac{N F_{2}-1}{G_{1}}+\frac{N F_{3}-1}{G_{1} G_{2}}+\frac{N F_{4}-1}{G_{1} G_{2} G_{3}}+\ldots .
$$

where $G_{m}$ is the single pass gain and $N F_{m}$ the noise figure of the $m^{\text {th }}$ section. From this equation it is apparent that the first few terms contribute most to the overall value of NF. Therefore if a lower overall noise figure is to be achieved, a low noise figure is necessary towards the input of the device. This implies a large carrier density at the input of the device. The optimum carrier density profile obtained by our simulation is presented in Fig. 1, this corresponds to a higher carrier density in the first few sections and a lower value at the output of the device. This profile was calculated for a $500-\mu \mathrm{m}$ long SOA biased at $250 \mathrm{~mA}$ with a power for the injected signal of $-50 \mathrm{dBm}$. In the same conditions a standard SOA provides a NF $20 \%$ larger.

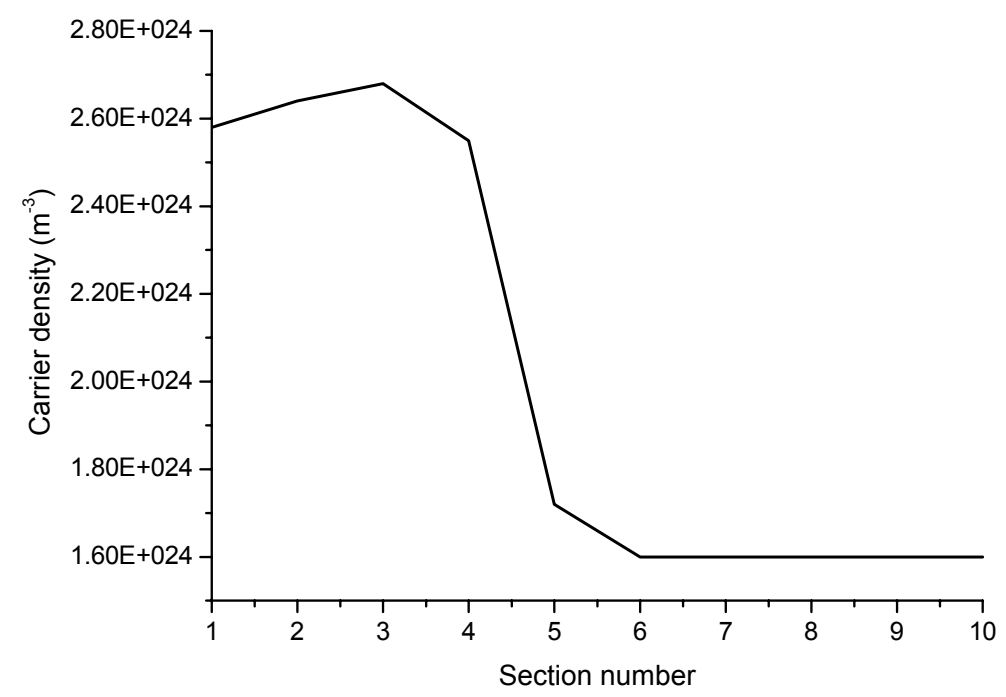

Figure 1. Ideal carrier density profile, $I_{\text {bias }}=250 \mathrm{~mA}$.

\section{DESIGN}

\subsection{Principle}

The aforementioned carrier profile may be achieved by several means, for instance through the use of a lateral lasing cavity placed at the output of the device controlling the carrier density at this position, to a lower level than that of the input. The cavity consists of two sets of mirrors placed outside the active waveguide, as shown on Figure 2. The mirrors consist of slots placed on both sides of the active layer. The material removed from the semiconductor material changes the value of the refractive index underneath, creating a spatial variation of the refractive index experienced by the field emitted. Constructive and destructive interferences occur and a particular wavelength is selected to experience a large reflectivity. The spontaneous emission coupled to the waveguide in the lateral direction will be trapped between the mirrors and amplified by the section of active material. Laterally the device acts as a laser, but longitudinally as an amplifier. The carrier density contributes to the gain of the laser. When the current supply reaches the threshold current, the carrier density is clamped at a density value where the gain provided by the semiconductor material equals the losses of the cavity. Hence it is possible by controlling the level of reflectivity of the mirror and the resonant wavelength to set the carrier density to a given value.

The design presented here provides an amplification path with lasing and non-lasing portions. The spontaneous emission (SE) is mostly generated and amplified by the non-lasing portion and only to a much lesser extent by the lasing portion. A reduction of the amplified spontaneous emission travelling from the output to the input and of the reflected amplified signal results in an automatic increase of the carrier density at the input section of the amplification path which reduces the NF of the input section and as a consequence lowers the overall NF of the SOA. In this way the ratio of amplification of the optical signal with respect to the ASE is controlled, such that the optical signal is preferentially amplified with respect to the ASE. 

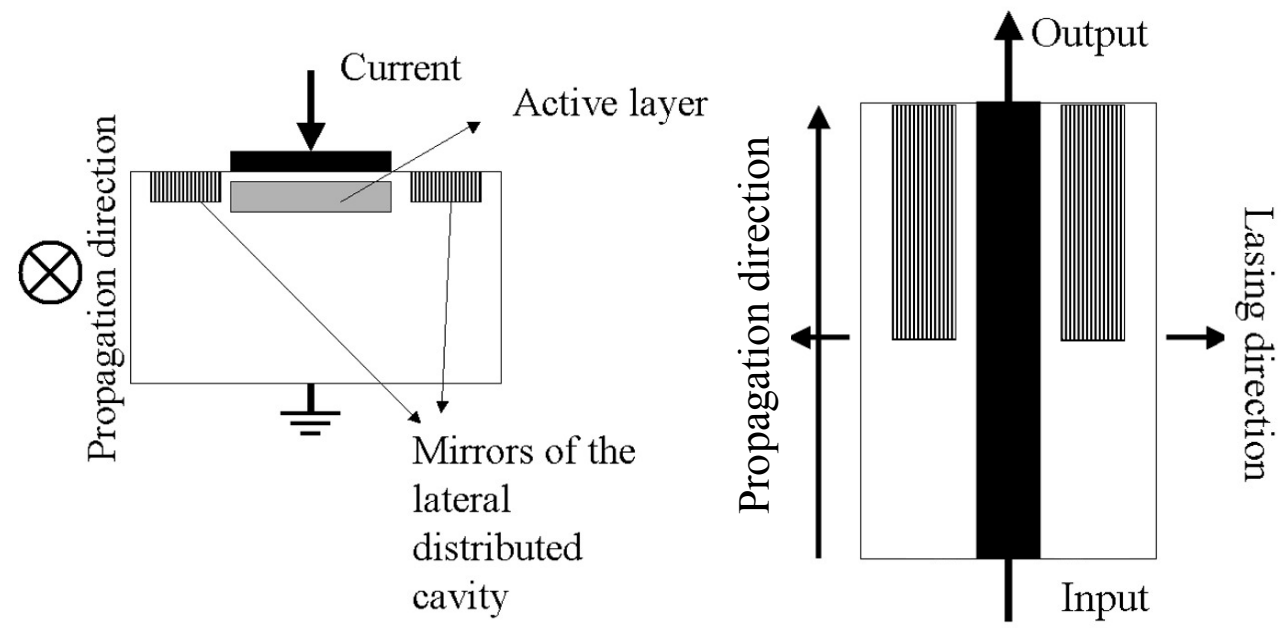

Figure. 2. Front and top view of the noise controlled SOA.

\subsection{Simulations}

Figure 3 shows the noise figure of the SOA as a function of the percentage of the lasing portion occupying the amplification path for a bias current of $250 \mathrm{~mA}$. The noise figure decreases with the length of the lasing portion and reaches a minimum around $60 \%$ before increasing rapidly. Similar results were found for different bias currents.

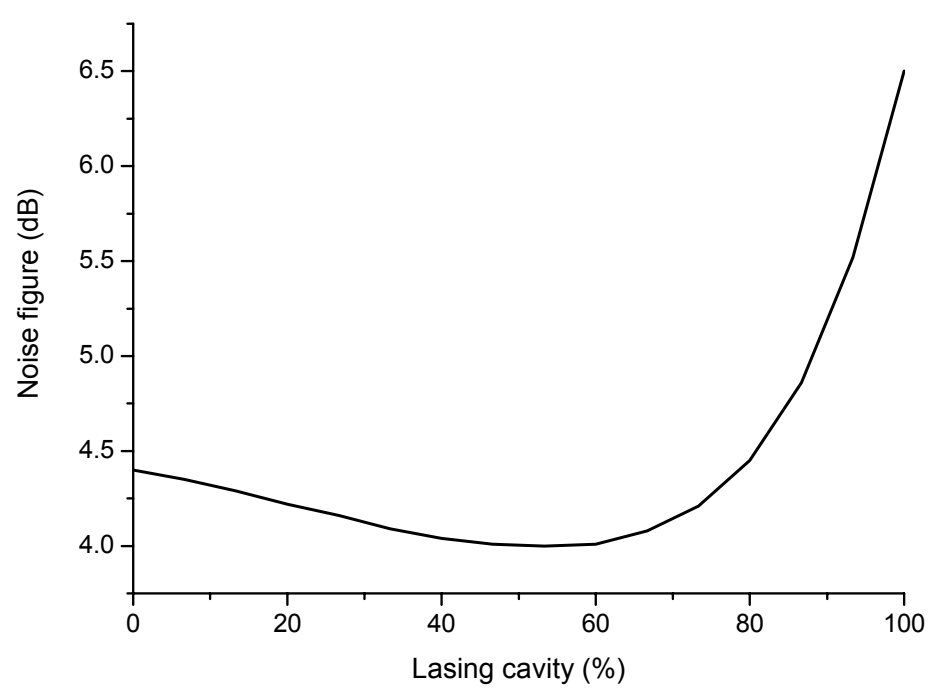

Figure. 3 Noise figure as a function of the length of the lasing cavity.

Simulations of the noise figure as a function of input power show that a lasing portion occupying $20 \%$ to $60 \%$ of the amplification path results in the lowest noise figure. Comparison between the best noise controlled SOA (60\% lasing portion) and the two extreme cases (no laser cavity and laser cavity along the full length of the active region) is shown on Figure 4 . The conventional SOA with no embedded lasing cavity $(0 \%$ conventional SOA) has a higher noise figure, by about $20 \%$, than SOAs with a lasing portion that occupies $60 \%$ of the amplification path up to an input power of about $-5 \mathrm{dBm}$. Above this value the performance of both devices are similar. A SOA with a lasing cavity occupying the full length of the amplification path (100\% linear optical amplifier) has the highest noise figure which is substantially independent of the injected power. The values of the pump current are given by way of example only, for simulation purposes, and it is not intended to limit the pump current to these particular values. 


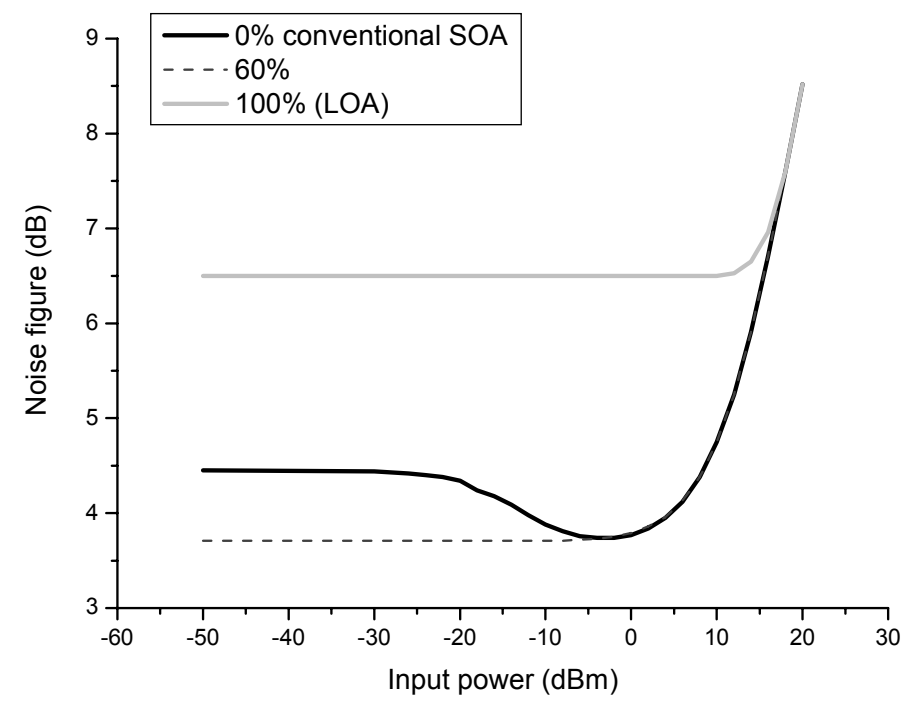

Figure. 4. Noise figure as a function of input power for different components.

\section{CONCLUSIONS}

Our model, based on a solution of the travelling wave description of the E-field in the amplifier cavity allows us to access the spatial dependence of the light and of the carrier density as well as calculating the resulting noise figure for a given device. From this it was shown that carefully engineering the carrier density distribution along the active region of the waveguide can improve the noise performance of SOAs. This can be achieved by introducing a lateral lasing cavity using mirrors consisting of slots on both sides of the amplification path. The optimal length of the lasing cavity was found to be $60 \%$ of the active region and simulations for this case show a reduction of the noise figure by $20 \%$, compared to a conventional SOA, thus demonstrating the potential of the technique.

\section{ACKNOWLEDGEMENTS}

Authors would like to thank Enterprise Ireland for its support under the technological development Program, project referenced CFDT/06/IT/332, and the INSPIRE Programme, funded by the Irish Government's Programme for Research in Third Level Institutions, Cycle 4, National Development Plan 2007-2013

\section{REFERENCES}

[1] S. Philippe, A. L. Bradley, B. Kennedy, F. Surre, and P. Landais: Experimental Investigation of Polarization Effects in Semiconductor Optical Amplifiers and Implications for All-Optical Switching, IEEE Journal of Lightwave Technology, vol. 26 (16), pp. 2977-2985, Aug. 15, 2008.

[2] S. Philippe, A. L. Bradley, R. Maldonado-Basilio, F. Surre, B. F. Kennedy, P. Landais, and a. H. SotoOrtiz: Polarization dependence of non-linear gain compression factor in semiconductor optical amplifier, Optics Express, vol. 16, no. 12, pp. 8641-8648, 2008.

[3] J. Mork, M. Nielsen, T. Berg: The Dynamics of Semiconductor Optical Amplifiers: Modeling and Applications, Optics and Photonics News, vol. 14, no. 7, pp. 42-48, July 2003.

[4] R. Brenot, F. Pommereau, O. Le Gouezigou, J. Landreau, F. Poingt, L. Le Gouezigou, B. Rousseau, F. Lelarge, F. Martin, G.H. Duan: Experimental study of the impact of optical confinement on saturation effects in SOA, Optical Fiber Communication Conference, Technical Digest, 2005, pp. 197-199.

[5] Patent number: US6731427 filled in 2004-05-04.

[6] T. Durhuus, B. Mikkelsen, K.E. Stubjkaer: Detailed dynamic model for semiconductor optical amplifiers and their crosstalk and intermodulation distortion, IEEE/OSA J. Lightwave Technology, vol. 10, no. 8, pp. 1056-1063, 1992. 